Pacific

Journal of

Mathematics

COVERING A CONVEX BODY BY ITS NEGATIVE HOMOTHETIC COPIES

Janusz Januszewski and Marek LassaK

Volume $197 \quad$ No. 1

January 2001 


\title{
COVERING A CONVEX BODY BY ITS NEGATIVE HOMOTHETIC COPIES
}

\author{
JANusz JANuszeWski AND MAREK LASSAK
}

\begin{abstract}
We estimate the number and ratio of negative homothetic copies of a $d$-dimensional convex body $C$ sufficient for the covering of $C$. If the number of those copies is not very large, then our estimates are better than recent estimates of Rogers and Zong. Particular attention is paid to the 2-dimensional case. It is proved that every planar convex body can be covered by two copies of ratio $-\frac{4}{3}$ (this ratio cannot be lessened if $C$ is a triangle).
\end{abstract}

Every convex body $C$ in Euclidean $d$-space $E^{d}$ can be covered by a homothetic copy of $C$ of ratio $-d$. This immediately follows from the papers of Neumann [10] for $d=2$, and Süss [12] in the general case. The covering by more than one negative homothetic copy was considered in $[\mathbf{3}],[\mathbf{9}]$ and [11]. The present paper establishes a few additional estimates about covering by negative copies. We also consider covering by negative and positive homothetic copies.

\section{Covering a $d$-dimensional body.}

Lemma. Let $P$ be a parallelotope of the smallest possible volume containing a convex body $C \subset E^{d}$. Denote by $\mathbf{v}_{1}, \ldots, \mathbf{v}_{d}$ the vectors determined by some $d$ edges of $P$ with a common origin. Let $\lambda_{1}, \ldots, \lambda_{d}$ be positive real numbers such that $\lambda_{1}+\cdots+\lambda_{d}=1$. The body $C$ contains a parallelotope $S$ whose $d$ edges with a common origin determine vectors $\lambda_{1} \mathbf{v}_{1}, \ldots, \lambda_{d} \mathbf{v}_{d}$.

Proof. From the considerations of $[4]$ it follows that for every $i \in\{1, \ldots, d\}$ there are boundary points $a_{i}$ and $b_{i}$ of $C$ such that $\overrightarrow{a_{i} b_{i}}=\mathbf{v}_{i}$. The required parallelotope $S$ has the $2^{d}$ vertices of the form $\lambda_{1} c_{1}+\cdots+\lambda_{d} c_{d}$, where $c_{i} \in\left\{a_{i}, b_{i}\right\}$ for $i=1, \ldots, d$.

By a box in $E^{d}$ we understand any set of the form

$$
\left\{\left(x_{1}, \ldots, x_{d}\right) ; r_{j} \leq x_{j} \leq s_{j} \text { for } j=1, \ldots, d\right\},
$$

where $r_{j}<s_{j}$ for $j=1, \ldots, d$. In particular, if $r_{1}=\cdots=r_{d}=0$ and $s_{1}=\cdots=s_{d}=1$, we obtain the unit cube $I^{d}$. 
Theorem 1. Assume that the d-dimensional unit cube $I^{d}$ can be covered by boxes $B_{1}, \ldots, B_{k}$ and denote by $p_{j m}$ the length of an edge of $B_{j}$ parallel to the $m$-th coordinate axis, where $j \in\{1 \ldots, k\}$ and $m \in\{1, \ldots, d\}$. Then every convex body $C \subset E^{d}$ can be covered by $k$ homothetic copies of $C$ whose homothety ratios are $r_{1}, \ldots, r_{k}$, where $\left|r_{j}\right|=p_{j 1}+\cdots+p_{j d}$ for $j=1, \ldots, k$.

Proof. Let $P$ denote a parallelotope of the smallest possible volume containing $C$. We do not make our considerations narrower by assuming that $P=I^{d}$ (if $P \neq I^{d}$, then we take an affine transformation $\tau$ such that $\tau(P)=I^{d}$ and we consider the body $C^{\prime}=\tau(C)$ instead of $\left.C\right)$. We apply the Lemma. For each $j \in\{1, \ldots, k\}$, take the numbers $\frac{p_{j 1}}{\left|r_{j}\right|}, \ldots, \frac{p_{j d}}{\left|r_{j}\right|}$ in place of $\lambda_{1}, \ldots, \lambda_{d}$, respectively. We see that for every $j \in\{1, \ldots, k\}$, the body $C$ contains a parallelotope $S_{j}$, whose $d$ independent edges determine vectors $\frac{p_{j 1} \mathbf{v}_{1}}{\left|r_{j}\right|}, \ldots, \frac{p_{j d} \mathbf{v}_{d}}{\left|r_{j}\right|}$. Hence for every $j \in\{1, \ldots, k\}$, the set $r_{j} S_{j}$ is a translate of $B_{j}$. Thus $B_{j}$ is a homothetic copy of $S_{j}$, where the ratio of the corresponding homothety $h_{j}$ is equal to $r_{j}$. Since $S_{j} \subset C \subset P$ for $j=1 \ldots, k$, we conclude that $C$ can be covered by homothetic copies $h_{1}(C), \ldots, h_{k}(C)$ of $C$.

The earlier mentioned covering by one copy of ratio $-d$ follows immediately from Theorem 1 by taking $k=1$ and $B_{1}=I^{d}$.

Consider two special cases of Theorem 1. Just put $k=2^{q}$, where $q \in$ $\{1, \ldots, d\}$, and $p_{1 m}=\cdots=p_{k m}=\frac{1}{2}$ for $m \leq q$ and $p_{1 m}=\cdots=p_{k m}=1$ for $m>q$. For the second special case take $k=t^{d}$ and $p_{j m}=\frac{1}{t}$ for all indexes, where $t \in\{1,2, \ldots\}$. We obtain the following corollary.

Corollary 1. Every convex body in $E^{d}$ can be covered by $2^{q}$ homothetic copies of ratio $-d+\frac{1}{2} q$ for every $q \in\{0,1, \ldots, d\}$. It can be also covered by $t^{d}$ homothetic copies of ratio $-\frac{d}{t}$ for every $t \in\{1,2, \ldots\}$.

A particular case of both statements of Corollary 1 is when we cover a convex body by $2^{d}$ homothetic copies of ratio $-\frac{1}{2} d$. Another particular case of the first statement is about covering by two homothetic copies of ratio $-d+\frac{1}{2}$.

Similarly, we can evaluate the homothety ratio for the covering by any particular number of negative copies (see Corollary 4 for such a general formula in $E^{2}$ ). For instance, every convex body in $E^{d}$ can be covered by 3 homothetic copies of ratio $-d+\frac{3}{4}$. This follows by taking $p_{11}=p_{21}=\frac{1}{2}$, $p_{12}=p_{22}=\frac{3}{4}, \quad p_{32}=\frac{1}{4}$, and $p_{i j}=1$ in remaining cases.

We conjecture that every convex body in $E^{d}$ can be covered by two negative homothetic copies of ratio $-d+1$ for $d$ odd, and of ratio $-d+1-\frac{1}{d+1}$ for $d$ even. Those values are attained for a $d$-dimensional simplex, as a simple but time consuming calculation shows. Let us present only a hint of how the two negative copies $S_{1}$ and $S_{2}$ are situated. If $d$ is odd, then $S_{2}$ is a 
translate of $S_{1}$ by vector $\frac{1}{d-1} \overrightarrow{a b}$, where $a$ and $b$ are the centroids of two opposite $\frac{d-1}{2}$-dimensional faces of $S_{1}$. If $d$ is even, then $S_{2}$ is a translate of $S_{1}$ by vector $\frac{1}{d} \overrightarrow{a b}$, where $a$ is the centroid of a $\frac{d}{2}$-dimensional face of $S_{1}$ and $b$ is the centroid of the opposite $\frac{d-2}{2}$-dimensional face.

The estimates of Corollary 1 can be also expressed in the following form, where $\lceil x\rceil$ means the smallest integer which is greater than or equal to $x$.

Corollary 2. Let $C \subset E^{d}$ be a convex body. If $-d \leq \lambda \leq-\frac{1}{2} d$, then some

$$
2^{\lceil 2 d+2 \lambda\rceil}
$$

homothetic copies of $C$ with ratio $\lambda$ cover $C$. If $-\frac{1}{2} d \leq \lambda<0$, then

$$
\left\lceil-\frac{d}{\lambda}\right\rceil^{d}
$$

homothetic copies of ratio $\lambda$ cover $C$.

If the number of equal negative homothetic copies is not very large, then the estimates (1) and (2) are better than the estimate

$$
\left(1-\frac{1}{\lambda}\right)^{d}(d \log d+d \log \log d+5 d), \quad \text { where } \quad d \geq 3,
$$

a special case of the formula (6) from the paper of Rogers and Zong [11]. It is easy to check that the estimates (1) and (2) remain better than (3), asymptotically as $d \rightarrow \infty$, for a polynomial number of negative covering copies. In other words, for $-\lambda$ of order $\log d$. A calculation shows that if $\lambda$ is sufficiently small and if $d \leq 8$, then (2) should be applied for obtaining better estimates than (3), and if $d \geq 9$, then (1) should be applied for this purpose.

Here is also a comparison of (2) with (3) for $d=3$. By (2), every 3dimensional convex body can be covered by $14^{3}=2744$ homothetic copies of ratio $-\frac{3}{14}=-0.2142 \ldots$, while by (3) we need 2815 such copies. For $d=3$ and $\lambda \leq-\frac{3}{14}$ formula (2) always gives fewer copies than (3), while (3) gives fewer copies for $\lambda>-\frac{3}{14}$.

Corollary 3. Every convex body in $E^{d}$ can be covered by $d^{d}+1$ homothetic copies of ratio $-1+d^{-d}(d+1)^{-1}$. Any desired number of those copies can be exchanged for copies of ratio $1-d^{-d}(d+1)^{-1}$.

Proof. Let $x_{k}=\frac{1}{d}-\frac{1}{d^{k}(d+1)}$ for $k=1, \ldots, d$, and $y_{k}=\frac{1}{d}+\frac{d-1}{d^{k}(d+1)}$ for $k=2, \ldots, d$. It is easy to check that $(d-1) x_{k}+y_{k}=1$ and that $(k-1) \frac{1}{d}+$ $x_{k}+y_{k+1}+\cdots+y_{d}=1-d^{-d}(d+1)^{-1}$.

In order to apply Theorem 1 , we will dissect the cube $I^{d}$ into $d^{d}+1$ convenient boxes. Here is how we provide the tiling. We represent $I^{d}$ as the 
union of $d$ horizontal strips of heights $x_{d}, \ldots, x_{d}, y_{d}$. We dissect each of the strips of height $x_{d}$ into $d^{d-1}$ boxes of successive widths $\frac{1}{d}, \ldots, \frac{1}{d}, x_{d}$.

At the second stage, the strip of height $y_{d}$ is dissected into $d$ strips by hyperplanes parallel to the $(d-1)$-st coordinate axis. The $(d-1)$-st widths of successive strips are $x_{d-1}, \ldots, x_{d-1}, y_{d-1}$. Each of the strips of the $(d-1)$-th width equal to $x_{d-1}$ is dissected into boxes of successive widths $\frac{1}{d}, \ldots, \frac{1}{d}, x_{d-1}, y_{d}$.

Similarly, we make tilings in successive stages. At the $(d-k+1)$-st stage we get $(d-1) d^{k}$ boxes of successive widths $\frac{1}{d}, \ldots, \frac{1}{d}, x_{k}, y_{k+1}, \ldots, y_{d}$.

At the last, $d$-th stage, we obtain $d-1$ boxes of successive widths $x_{1}, y_{2}$, $\ldots, y_{d}$. The total number of boxes so obtained is $(d-1) d^{d-1}+(d-1) d^{d-2}+$ $\cdots+(d-1) d+d+1=d^{d}+1$. From the equalities at the beginning of the proof we see that the sum of the lengths of the independent edges of each box is $1-d^{-d}(d+1)^{-1}$. Thus from Theorem 1 we obtain the claim of Corollary 3.

In particular, when all the copies in Corollary 3 are positive, we obtain an estimate for the well known problem of Hadwiger [5] which asks if every convex body $C \subset E^{d}$ can be covered by $2^{d}$ smaller positive homothetic copies. For $d=3$ we know only some estimates of the number of those copies $C$, see [7], [8] and [11]. For $d \geq 3$ the estimate of the number of copies of positive ratio smaller than 1 presented in Corollary 3 is better than the estimate from [7], but for $d=3$ it is weaker than that from [8], and for $d \geq 6$ it is weaker than the estimate $\left(\begin{array}{c}2 d \\ d\end{array}\right)(d \log d+d \log \log d+5 d)$ presented in [2], [11] and [13]. Thus here we get the best estimates 257 in $E^{4}$ and 3126 in $E^{5}$. The advantage of the estimate of Corollary 3 is that we have a universal ratio of homothety. Remember that such estimates with a universal homothety ratio were known only for $d \leq 3$ : Every 2-dimensional convex body can be covered by 4 copies of ratio $\sqrt{2} / 2$ (see [6]), and every 3 dimensional convex body can be covered by 24 copies of a universal positive ratio smaller than 1 (see $[8]$ ).

\section{Covering a two-dimensional body.}

Observe that every positive integer $n$ can be represented either in the form $n=m^{2}+k$, where $m$ and $k$ are positive integers such that $0 \leq k \leq m-1$, or in the form $n=m(m+1)+k$, where $m$ and $k$ are positive integers such that $0 \leq k \leq m$.

Corollary 4. Every convex body in $E^{2}$ can be covered by $n$ homothetic copies of ratio $\frac{-2 m^{2}-2 m+k}{m^{2}(m+1)}$ provided $n=m^{2}+k$, where $0 \leq k \leq m-1$, and of ratio $\frac{-2 m^{2}-3 m+k-1}{m(m+1)^{2}}$ provided $n=m(m+1)+k$, where $0 \leq k \leq m$. Any desired number of those copies can be exchanged for copies with ratio of the opposite sign. 
Figure 1 shows the idea of Corollary 4 and how it results from Theorem 1. We consider here only $n$ fulfilling $2^{2} \leq n \leq 3^{2}$ in order to fix our attention. We see how homothety ratios $-1,-\frac{11}{12},-\frac{5}{6},-\frac{7}{9},-\frac{13}{18},-\frac{2}{3}$ are obtained for $n=4, \ldots, 9$, respectively.

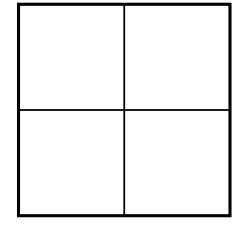

$\frac{1}{2}+\frac{1}{2}=1$

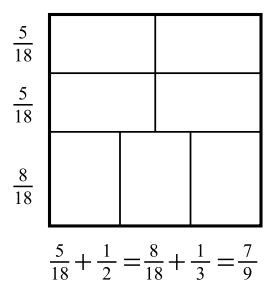

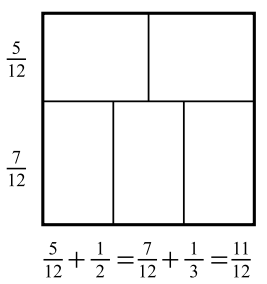

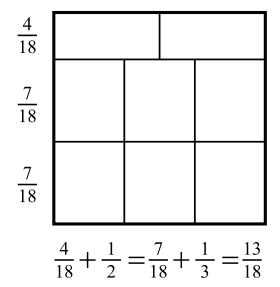

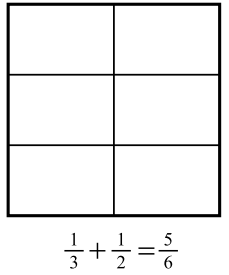

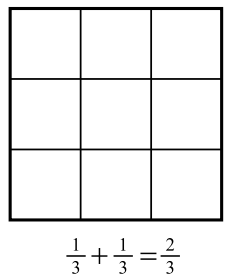

Figure 1.

We conjecture that a covering by 5 copies of ratio $-\frac{2}{3}$ is always possible (this value cannot be improved for a triangle). Better estimates than corresponding estimates for $n=2,3$ and 4 are obtained in [3] and [9]. They are $-\sqrt{2}$ for 2 copies, -1 for 3 copies (this ratio cannot be improved, as the example of a triangle shows), and less than -1 for 4 copies. Recall the conjecture from [9] that every planar convex body can be covered by 4 copies of ratio $-\frac{4}{5}$. Below we present improvements of the estimates for covering by 2 and by 7 negative copies. The example of a triangle shows that the following estimate $-\frac{4}{3}$ for covering by two copies is the best possible. Let us add that the estimate was conjectured in [9].

Theorem 2. Every convex body $C \subset E^{2}$ can be covered by two homothetic copies of ratio $-\frac{4}{3}$.

Proof. Let $C \subset E^{2}$ be a convex body. Let $c d e$ be a triangle contained in $C$ with the greatest possible area. In order to simplify further computations, we will make some convenient assumptions. Since the affine image of this triangle is a triangle of maximum area in the corresponding transformed body, we loose no generality in assuming that $c=c(-1,0), d=d(1,0), e=$ $e(0,1)$. As usual, the numbers in brackets denote the coordinates of a given point. The triangle with vertices $t_{1}(0,-1), t_{2}(2,1), t_{3}(-2,1)$, contains $C$ (see 
Fig. 2). The reason is that the vertices of the triangle $c d e$ are in the sides of the triangle $t_{1} t_{2} t_{3}$ which has parallel sides (thus a point of $C$ outside $t_{1} t_{2} t_{3}$ would permit the construction of a triangle of a greater area in $C$ ). Denote by $o$ the centroid of the triangle $c d e$. Let $p_{1}, p_{2}, p_{3}$ be the boundary points of $C$ on the segments $o t_{1}, o t_{2}, o t_{3}$, respectively. Without loss of generality we can also assume that $\left|o t_{3}\right| /\left|o p_{3}\right| \geq\left|o t_{1}\right| /\left|o p_{1}\right|$ and $\left|o t_{3}\right| /\left|o p_{3}\right| \geq\left|o t_{2}\right| /\left|o p_{2}\right|$. If this assumption is not satisfied, we can apply an affine transformation which changes the order of the vertices $c, d, e$.

In order to shorten further explanations, we introduce the following notation. A homothetic copy of a set with the homothety ratio $-\frac{4}{3}$ will be called a copy. We say that a point is on the left (on the right) of a non-horizontal line $L$ if its first coordinate is not greater (not smaller) than the first coordinate of the corresponding point of $L$ on the same horizontal level. If a point is denoted by a symbol, then its first and second coordinates are denoted by $x$ and $y$ with just this symbol as the index.

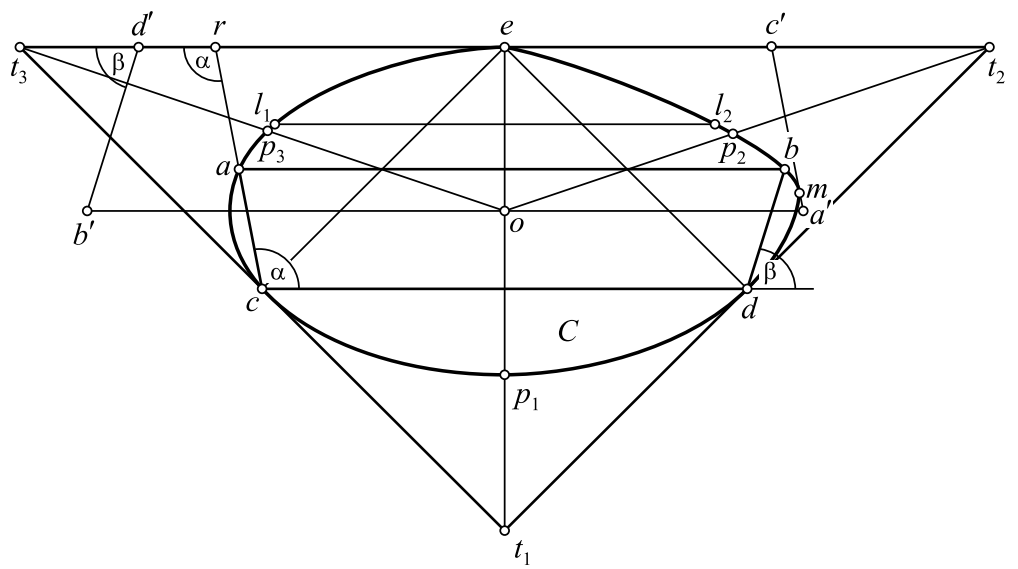

Figure 2.

Denote by $C_{1}, C_{2}$ and $C_{3}$ those parts of $C$ whose points $(x, y)$ fulfill the inequalities $y \leq \frac{1}{3}, \quad \frac{1}{3} \leq y \leq \frac{2}{3}$ and $\frac{2}{3} \leq y$, respectively. Observe that the part of the triangle $t_{1} t_{2} t_{3}$ whose points $(x, y)$ fulfill the inequality $y \leq \frac{1}{3}$ can be covered by one copy of the triangle $c d e$. Thus $C_{1}$ can be covered by a copy of $C$. In order to prove that another copy of $C$ is able to cover $C_{2} \cup C_{3}$, it is sufficient to show that $C_{2} \cup C_{3}$ can be covered by a copy of the trapezoid $c d b a$, where $a\left(x_{a}, \frac{1}{2}\right)$ and $b\left(x_{b}, \frac{1}{2}\right)$ (with $x_{a}<x_{b}$ ) are points on the boundary of $C$ (see Fig. 2). The required copy is the trapezoid $T=c^{\prime} d^{\prime} b^{\prime} a^{\prime}$, where the copy of the segment $c d$ is the segment $d^{\prime} c^{\prime}$ contained in the line $y=1$, such that all the points of $C$ are on the left of the straight line containing $a^{\prime} c^{\prime}$, 
and such that a boundary point $m\left(x_{m}, y_{m}\right)$ of $C$ belongs to the segment $a^{\prime} c^{\prime}$. Obviously, $-\frac{3}{2} \leq x_{a} \leq-\frac{1}{2}$ and $\frac{1}{2} \leq x_{b} \leq \frac{3}{2}$.

Let $l_{1}, l_{2}$ be the boundary points of $C$ on the line $y=\frac{2}{3}$ (see Fig. 2). We have $\left|l_{1} l_{2}\right| \leq 2$. Here is why. If $y_{p_{1}} \geq-\frac{1}{3}$, then $x_{p_{2}} \leq 1$ and $x_{p_{3}} \geq-1$ which implies $\left|l_{1} l_{2}\right| \leq 2$. Also $y_{p_{1}}<-\frac{1}{3}$ gives $\left|l_{1} l_{2}\right| \leq 2$ since the opposite leads to the conclusion that the area of the triangle $l_{1} l_{2} p_{1}$ is greater than the area of the triangle $c d e$.

Case 1: When $y_{m} \leq \frac{2}{3}$.

First we show that $C_{2} \subset T$.

Take the point $s\left(x_{s}, \frac{1}{3}\right)$ on the straight line through $a$ and $e$ and the point $t\left(x_{t}, \frac{1}{3}\right)$ on the straight line through $b$ and $e$. Let $b^{-} a^{-} c^{-} d^{-}$be the copy of the trapezoid bacd with $a^{-}=t$ and $b^{-}=s$. The trapezoid $b^{-} a^{-} c^{-} d^{-}$ covers $C_{2}$. Obviously, $x_{m} \leq x_{t}$. Consequently, $C_{2} \subset T$.

Now we show that $C_{3} \subset T$.

Denote by $\alpha$ the angle $\angle a c d$ and let $\beta=180^{\circ}-\angle c d b$. We can assume that $\alpha \geq \beta$. The reason is that if $\alpha<\beta$ and if $C_{3}$ is not a subset of $T$, then the convexity of $C$ implies that $a \notin T$, a contradiction to the inclusion $C_{2} \subset T$.

Take the point $r\left(x_{r}, 1\right)$ on the straight line through $a$ and $c$. Since $\alpha \geq \beta$, in order to show the inclusion $C_{3} \subset T$, it is sufficient to show that $x_{r} \geq x_{d^{\prime}}$ (see Fig. 2). The rest of Case 1 is devoted to this aim.

We omit an elementary calculation which gives $x_{m} \leq \frac{5}{3}$ and

$$
x_{r}-x_{d^{\prime}}=\frac{5}{3}+\left(2 x_{a}+2\right) y_{m}-x_{m} .
$$

If $x_{a}>-1$, then $x_{r}-x_{d^{\prime}} \geq \frac{5}{3}+\left(2 x_{a}+2\right) y_{m}-\frac{5}{3}>0$. Thus we need consider only the case when $x_{a} \leq-1$.

First assume that $x_{b}<1$. If $y_{m} \leq \frac{1}{2}$, then $x_{m} \leq x_{t}=\frac{4}{3} x_{b}$. Moreover, $m$ is on the left of the straight line through $e$ and $b$. Thus, $y_{m} \leq 1-\frac{x_{m}}{2 x_{b}}$ and by (4) we get $x_{r}-x_{d^{\prime}} \geq \frac{5}{3}+\left(2 x_{a}+2\right)\left(1-\frac{x_{m}}{2 x_{b}}\right)-x_{m} \geq \frac{2}{3} x_{a}-\frac{4}{3} x_{b}+\frac{7}{3}>0$. If $y_{m}>\frac{1}{2}$, then $x_{m} \leq x_{b}<1$. Putting $x_{m}=1, y_{m}=\frac{2}{3}$ and $x_{a}=-\frac{3}{2}$ in (4) we obtain $x_{r}-x_{d^{\prime}}>0$.

Next assume that $x_{b} \geq 1$. Consider three subcases. Subcase 1: When $\frac{1}{2}<y_{m} \leq \frac{2}{3}$.

Assume that $C_{3}$ is not a subset of $T$. Then the point $u\left(x_{u}, \frac{2}{3}\right)$ from the segment $c d^{\prime}$ belongs to $C$. The point $m$ is on the right of the straight line parallel to the segment $a c$ and passing through $b$, since otherwise we have the false conclusion that $b \notin T$. Thus $x_{m} \geq\left(y_{m}-\frac{1}{2}\right)\left(2 x_{a}+2\right)+x_{b}$. Of course, the point $v\left(x_{v}, \frac{2}{3}\right)$ from the segment $\mathrm{em}$ belongs to $C$. We obtain

$$
|u v|=\frac{x_{m}}{3-3 y_{m}}-\frac{4}{3} x_{a}+\frac{4}{3} y_{m}\left(x_{a}+1\right)-\frac{2}{3} x_{m}+\frac{7}{9} .
$$


Taking $\left(y_{m}-\frac{1}{2}\right)\left(2 x_{a}+2\right)+x_{b}$ in place of $x_{m}$ and $\frac{1}{2}$ in place of $y_{m}$ we obtain $|u v|>-\frac{2}{3} x_{a}+\frac{13}{9}>2$. Since $u$ and $v$ are points on the segment $\left[l_{1}, l_{2}\right]$, we obtain a contradiction to the inequality $\left|l_{1} l_{2}\right| \leq 2$ shown before starting Case 1.

Subcase 2: When $\frac{1}{3} \leq y_{m} \leq \frac{1}{2}$ and when $x_{a}<\frac{1}{2} x_{b}^{2}-\frac{2}{3} x_{b}-\frac{23}{24}$.

Assume that $C_{3}$ is not a subset of $T$. The point $w\left(\frac{2}{3} x_{b}, \frac{2}{3}\right)$ from the segment $e b$ belongs to $C$. Observe, that $|u w|=\frac{2}{3} x_{b}-\frac{4}{3} x_{a}+\frac{4}{3} y_{m}\left(x_{a}+1\right)-$ $\frac{2}{3} x_{m}+\frac{7}{9}$. The point $m$ is on the left of the straight line passing through $e$ and $b$. Thus $y_{m} \leq 1-\frac{x_{m}}{2 x_{b}}$. Let $q\left(x_{q}, y_{q}\right)$ be the common point of the straight lines containing the segments $e b$ and $d t_{2}$. We get $x_{m} \leq x_{q}=\frac{4 x_{b}}{2 x_{b}+1}$, which together with our assumption $x_{a}<\frac{1}{2} x_{b}^{2}-\frac{2}{3} x_{b}-\frac{23}{24}$ leads to the false inequality

$$
|u w| \geq \frac{12 x_{b}^{2}-16 x_{b}-24 x_{a}-23}{18 x_{b}+9}+2>2 .
$$

Subcase 3: When $\frac{1}{3} \leq y_{m} \leq \frac{1}{2}$ and when $x_{a} \geq \frac{1}{2} x_{b}^{2}-\frac{2}{3} x_{b}-\frac{23}{24}$.

Taking $y_{m}=\frac{1}{2}, x_{m}=x_{q}$ and $x_{a}=\frac{1}{2} x_{b}^{2}-\frac{2}{3} x_{b}-\frac{23}{24}$ in (4) we obtain that $x_{r}-x_{d^{\prime}}>0$.

Case 2: When $y_{m}>\frac{2}{3}$.

The proof of the inclusion $C_{2} \subset T$ is similar to Case 1 .

Finally, we show that $C_{3} \subset T$.

Assume the contrary. If $\alpha<\beta$, then $a \notin T$, a contradiction. Let $\alpha \geq \beta$. Obviously the point $z\left(x_{z}, \frac{2}{3}\right) \in m d$ belongs to $C$. Observe that $u \in C$. We have

$$
|u z|=\frac{2}{3} x_{m}\left(\frac{1}{y_{m}}-1\right)-\frac{2}{3 y_{m}}-\frac{4}{3} x_{a}+\frac{4}{3} y_{m}\left(x_{a}+1\right)+\frac{16}{9} .
$$

Of course, $m$ is on the right of the straight line by $e$ and $d$. Thus, $x_{m} \geq$ $1-y_{m}$. Taking $x_{m}=1-y_{m}, x_{a}=-\frac{1}{2}$ and $y_{m}=\frac{2}{3}$ in (5) we get $|u z|>2$, a contradiction.

The following estimate $-\frac{2}{3}$ is better than the estimate $-\frac{7}{9}$ resulting from Corollary 4. We conjecture that the best possible ratio here is $-\frac{10}{17}=$ $0.5882 \ldots$ It is easy to show that a triangle can be covered by 7 copies of ratio $-\frac{10}{17}$ and it cannot be covered if the negative ratio is over $-\frac{10}{17}$.

Proposition. Every convex body $C \subset E^{2}$ can be covered by 7 homothetic copies of ratio $-\frac{2}{3}$.

Proof. We can inscribe an affine-regular hexagon $H=$ abcdef in $C$ (see [1]). Three of the lines containing the sides of $H$ bound a triangle $T_{1}$ containing $H$ and the other three a triangle $T_{2}$ containing $H$. Since $H$ is inscribed in $C$, we see that $C \subset T_{1} \cup T_{2}$. We can assume that the center of symmetry of $H$ is the origin $o$ of $E^{2}$. Of course, $-\frac{2}{3} H \subset-\frac{2}{3} C$. Thus in order to show 
the promised estimate, it is sufficient to cover $T_{1} \cup T_{2}$ by 7 translates of $-\frac{2}{3} H$. Observe that these are $-\frac{2}{3} H$ and its translates by vectors $2 \overrightarrow{\mathrm{om}}_{i}$ for $i=1, \ldots, 6$, where $m_{1}, \ldots, m_{6}$ are midpoints of the sides of $-\frac{2}{3} H$.

\section{References}

[1] A.S. Besicovitch, Measure of asymmetry of convex curves, J. London. Math. Soc., 23 (1948), 237-240.

[2] V. Boltyanski, H. Martini and P.S. Soltan, Excursions into Combinatorial Geometry, Springer, 1997.

[3] G.D. Chakerian and S.K. Stein, On measures of symmetry of convex bodies, Canad. J. Math., 17 (1965), 497-504.

[4] Some intersection properties of convex bodies, Proc. Amer. Math. Soc., 18 (1967), 109-112.

[5] H. Hadwiger, Ungelöste Probleme, Nr. 20, Elem. Math., 12 (1957), 121.

[6] M. Lassak, Covering a plane convex body by four homothetical copies with the smallest positive ratio, Geom. Dedicata, 21 (1986), 151-167.

[7] _ Covering the boundary of a convex set by tiles, Proc. Amer. Math. Soc., 104 (1988), 269-272.

[8] _ Covering a three-dimensional convex body by smaller homothetic copies, Beitr. Algebra Geom., 39 (1998), 259-262.

[9] M. Lassak and É. Vásárhelyi, Covering a plane convex body with negative homothetical copies, Stud. Sci. Math. Hung., 28 (1993), 375-378.

[10] B.H. Neumann, On some affine invariants of closed convex regions, J. London Math. Soc., 14 (1939), 262-272.

[11] C.A. Rogers and C. Zong, Covering convex bodies by translates of convex bodies, Mathematika, 44 (1997), 215-218.

[12] W. Süss, Über eine Affininvariante von Eibereichen, Arch. Math., 1 (1948), 127-128.

[13] L. Szabo, Recent results on illumination problems, Bolyai Society Mathematical Studies, 6 (1997), 207-221.

Received March 1, 1999.

Instytut Matematyki I Fizyki Atr

85-796 BydGOSZCZ

POLAND

E-mail address: januszew@atr.bydgoszcz.pl

Instytut Matematyki I Fizyki Atr

85-796 BydgOSZCZ

POLAND

E-mail address: lassak@atr.bydgoszcz.pl 